\title{
Review of: "Nitric oxide negatively regulates gibberellin signaling to coordinate growth and salt tolerance in Arabidopsis"
}

xiaoying zhao

1 Hunan University

Potential competing interests: The author(s) declared that no potential competing interests exist.

In the present study, the authors provide a new and important mechanism by which NO negatively regulates gibberellin signaling to coordinate growth and salt tolerance in Arabidopsis. The authors carried out a series of biochemical, molecular and genetic studies, and demonstrate that S-nitrosylation of Cys-374 in the RGA protein inhibits its interaction with the F-box protein SLY1, thereby preventing its proteasomal degradation under salinity condition, which in turn retards growth and enhances salt tolerance. The experiments were well organized in general and the results are of significant novelty.

I have two suggestions that I'd like the authors to take into consideration:

1. In figure $2 F$, the fluorescence signal for the RGA-SLY1 interaction should be quantified.

2. The authors showed that GSNO treatment reduced the RGA-SLY1 interaction in pull-down and BiFC assays (Figure $2 \mathrm{E}, \mathrm{F})$. Nevertheless, this interaction needs to be examined under more physiological conditions. The strength of the RGA-SLY1 interaction should be examined in thegsnor1-3 or nox1 background in which NO over-accumulated. 